\title{
Analysis of Muscle and Metabolic Activity during Multiplanar-Cardiofitness Training
}

\author{
Arrigo Palumbo, Teresa Iona, Vera Gramigna, Antonio Ammendolia, \\ Maurizio Iocco, and Gionata Fragomeni \\ Magna Graecia University, Campus S. Venuta, \\ 88100 Catanzaro, Italy \\ \{palumbo, iona, gramigna, ammendolia, miocco, \\ fragomeni\}@unicz.it
}

\begin{abstract}
Electronic devices have been useful to evaluate muscle fatigue and activation, co-ordination and metabolic consuption among different sports activities. Since these important variables have not been investigated during fitness training, the present study aims to analyze the activity of the major muscles of the lower extremity during training activities on a cardiofitness apparatus (Cardio Wave ${ }^{\mathrm{TM}}$, Technogym ${ }^{\circledR}$ - Gambettole, Italy). This device is able to stimulate the multiplanar movements of lower limbs by combining various types of movement according to the physical principles of human motion. Working simultaneously on three axes by a sliding movement of lower limbs should activate different muscle groups following four positions at different intensity level. Muscles activity and training effectiveness were evaluated by monitoring Electromiography signal, metabolic data, oxygen uptake and heart rate. The goal of this research is to develop a system able to manage different information coming by varius electronic devices.
\end{abstract}

Keywords: EMG, Cardiofitness, Wavelet.

\section{Introduction}

More and more people are deciding to start exercising in a fitness center in order to increase muscle strength and reduce body fat. Several models of cardiofitness machines are available to practitioners who freely choose the exercise modality. However, incorrect exercise intensities and positions are related to inefficacious training and incidence of injuries 1 ..

Cardio $\mathrm{Wave}^{\mathrm{TM}}(\mathrm{CW})$ is a cardiofitness apparatus specifically designed to allow movements along three axes (Fig. 1) by combining different movements which extensively train lower limb muscles according to four positions:

- Basic (B): with chest erect.

- Intermediate (I): with chest leant forward.

- Advanced (A): with chest and hands leant forward the ground.

- Free style (F): with arms free to oscillate.

Each position is meant to train different muscle groups. 


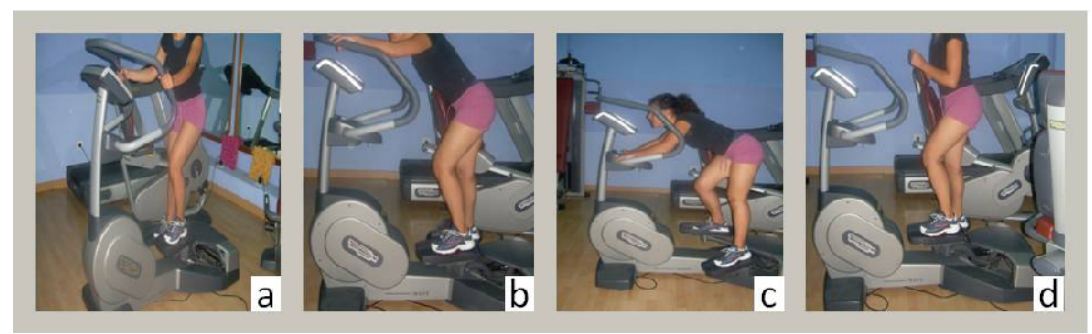

Fig. 1. Cardio Wave device positions: a) Standard , b) Advanced, c) Intermediate, d) Freestyle

During whatever sport activity fatigue is indicated by a decrease of muscle strength and power. Most studies of neuromuscular activity and fatigue have evaluated isometric and cyclical contractions. However, these types of contractions may not be representative of muscle activity and fatigue during physical exercise 2.. Indeed, available data suggest that the development of fatigue is specific to contraction type, intensity and duration of activity 3.. An exercise of the type that occurs during a fitness training session is characterized by a variety of muscle activities.

In order to investigate lower limb muscle activity and training optimization at the four positions (i.e. B, I, A, F) and in the 3 intensity levels, several parameters were monitored. Particularly Electromyography (EMG), Hearth Rate (HR), Oxygen uptake (VO2) and Lactate threshold (LA) were recorded by means of four different devices.

EMG was used to display muscle activation patterns and interpret both dysfunction and functional muscle recruitment $4 . . \mathrm{HR}$ is an important parameter to evaluate a specific exercise task in terms of the strain it places on the individual's aerobic system 5.. Both LA threshold and VO2 are predictors of aerobic exercise capacity 6 .

Aim of this research was to evaluate training activities using the information coming from different electronic devices.

\section{Methods}

\subsection{Subjects}

During a test session nine healthy subjects - 5 trained and 4 untrained - with no history of musculoskeletal injuries and free from cardiac or metabolic disorders were recruited. They agreed to participate in the study. All subjects were selected on a voluntary basis from a fitness center population. They represented a wide spectrum of body weight, height, age and muscle strength. They ranged in age between 25 and 33 years with a mean value of 27.4 years. Their weight ranged from $65.4 \mathrm{~kg}$ to $99.8 \mathrm{~kg}$ with a mean value of $75.9 \mathrm{~kg}$. Their height ranged from $167 \mathrm{~cm}$ to $177.8 \mathrm{~cm}$ with a mean value of $171.5 \mathrm{~cm}$.

We considered as trained group the subjects that claimed to train at least twice a week at a moderate intensity $\left(60-70 \% \mathrm{HR}_{\max }\right)$, for a minimum duration of 45 minutes / session.

Before training sessions all groups performed a 20-minute stretching protocol for lower limbs 7. for 30 seconds. This exercise was repeated 3 times for each muscle 
group. After a 10-minute warming-up on the ergometer, subjects were asked to perform randomly in all the four positions allowed by $\mathrm{CW}$.

\subsection{Data Recording}

Commercial electronic devices are used to monitor different physics parameters for subject recruited and for each training session (Table 1).

Vastus medialis (VM), rectus femoris (RF) and biceps femoris (BF) muscles were selected for EMG analysis, as they are the most stimulated muscles in this kind of exercise.

The subjects were prepared for placement of EMG electrodes: the skin of each electrode site was shaved and cleaned carefully with alcohol in order to have a low inter-electrode resistance. The activity of each muscle was recorded by means of a couple of electrodes (Ag-Ag/Cl; Aurion s.r.l., Milan, Italy; $20 \mathrm{~mm}$ diameter, $20 \mathrm{~mm}$ inter-electrode distance). The electrodes were placed longitudinally on the motor point areas of the muscle examined. EMG signals were recorded telemetrically and the electrodes were placed on the RF, mid-way between the anterior superior iliac spine and the superior border of the patella; the BF over the long head, half-way between the ischial tuberosity and lateral femoral epicondyle; the VM at $80 \%$ on the line between the anterior spina iliaca and the joint space in front of the anterior border of the medial ligament 8 ..

To reduce movement artefacts, the electrodes were taped to the skin with an elastic bandage. Moreover, the usage of a wireless device allowed not to interfere with the subject's movements. EMG activity was acquired by using a wireless low power signal conditioning electronics device (ZeroWire Aurion s.r.l., Milan, Italy), in order to achieve both a stable input impedance and a high value of signal amplification (up to $100,000 \mathrm{~V} / \mathrm{V})$; a $2 \mathrm{KHz}$ sample frequency was used for analog- to -digital conversion.

$\mathrm{VO}_{2}, \mathrm{HR}$ and La concentrations were measured at rest and during exercise and recovery 9,10 ..

During the test session, $\mathrm{VO}_{2}$ was monitored continuously using a smaller portable gas analyzer (FitmatePro ${ }^{\mathrm{TM}}$, Cosmed, Rome, Italy), in order to assess cardio respiratory function. This device allowed the direct measurement of $\mathrm{VO}_{2}$ max during an up to maximal exertion incremental exercise protocol, the extrapolation of $\mathrm{VO}_{2} \max$ during a sub-maximal incremental exercise, and the Estimation of $\mathrm{VO}_{2} \max$ based on the results achieved during some standard field tests.

Heart Rate was monitored by telemetric heart-rate bands (Polar, Kempele, Finland) with the registration of values during each minute of exercise on $\mathrm{CW}$. We considered the last minute for statistical purposes 11-13.. The $4.0 \mathrm{mmol} / \mathrm{l} \mathrm{La}$ threshold was measured with a lactate-meter (Accu-check, Roche Diagnostic, France) at rest and during exercise (at the end of each 3-minutes step) and recovery (3, 6, 9 minutes after performing the test).

All recording data coming from different devices were independently collected, were stored in a common database and processed togheter with a custom-written software in order to obtain information useful to a training activity analysis. Data coming from different devices use a storage memory of about 300 MegaByte for any subject. The computational time necessary to complete one test session is about 2 hours for any participant. 
Table 1. Evaluated parameters during exercise

\begin{tabular}{llllll}
\hline Time (min:s) & Intensity (RPM) & \multicolumn{4}{l}{ Parameter recording } \\
\hline & & & & & \\
$00: 00$ & 120 & EMG & $\mathrm{VO}_{2}$ & & $\mathrm{HR}$ \\
$04: 00$ & 200 & EMG & $\mathrm{VO}_{2}$ & LA & HR \\
$08: 00$ & 300 & EMG & $\mathrm{VO}_{2}$ & LA & HR \\
$12: 00$ & STOP & & & LA & HR \\
15.00 & STOP & & & LA & \\
$18: 00$ & STOP & & & LA & \\
$21: 00$ & STOP & & & LA & \\
\hline
\end{tabular}

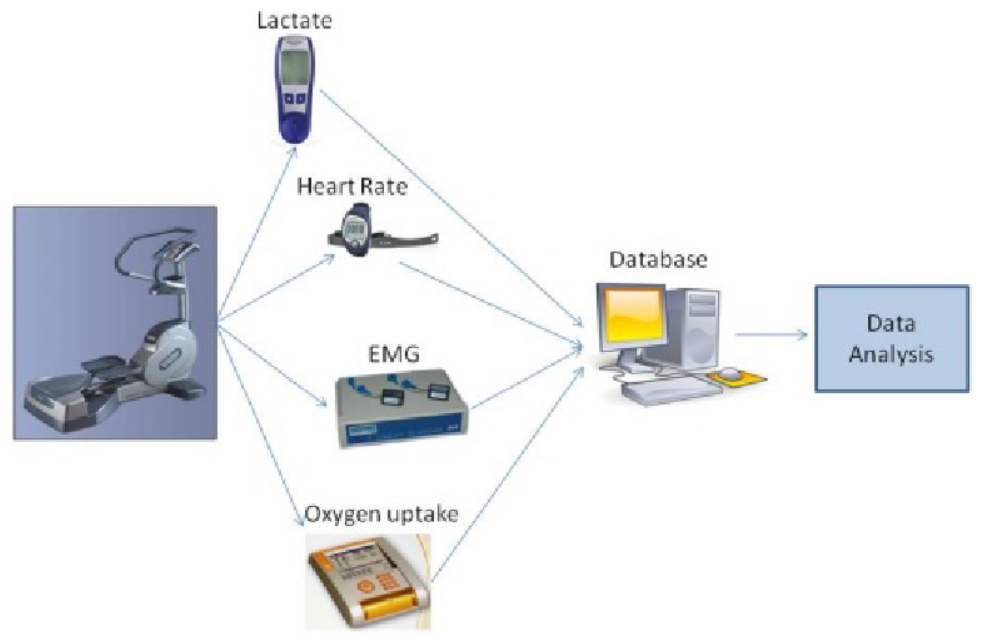

Fig. 2. Data flow

\subsection{Fatiguing Protocol}

We used a fitness-specific intermittent exercise protocol, which provided a fatiguing activity. Each subject performed 4 test sessions, one for each $\mathrm{CW}$ position (i.e., B, I, A, F). During a test session, subjects randomly performed the same 12-minute exercise at different intensities (4-minute step at three different intensities: low - 120 RPM, middle - 200 RPM and high intensity -300 RPM) imposed by the CW machine in the same position. In each session, subjects warmed up by $4 \mathrm{~min} / \mathrm{step}$ at the three intensities selected; each session was separated by $180 \mathrm{~min}$ of recovery, but the subjects could only make 2 sessions per day in order to aid muscle fatigue recovery.

\subsection{Data Processing}

In order to test the different parameters for the purpose of evaluating muscle activity and fatigue, EMG data were analyzed by using LabView 8.0 software (National Instruments corp., Austin, TX, USA). 
Continuous Wavelet Transform (CWT) was used in order to identify particular time-frequency signal models. Wavelet analysis is becoming a common tool for studying the localized variations of power within a time series. By decomposing a time series into time-frequency space, it is possible to evaluate both the dominant modes of variability and how those modes vary in time 14,15 .

In particular, in order to analyze EMG signals, Mean Instantaneous Frequency (MIF) was calculated. Starting from the spectrographic analysis of the signal, we aimed at pointing out how the signal's central frequency changes in time. MIF represents the power of spectral response for each subject studied. MIF is a mean value calculated to get a group parameter aimed at estimating how much the strength of muscle contraction and the degree of muscle fatigue vary in time 15.. MIF represents power spectral response for each subject trial: from MIF vector a mean value is calculated in order to obtain a clustering parameter.

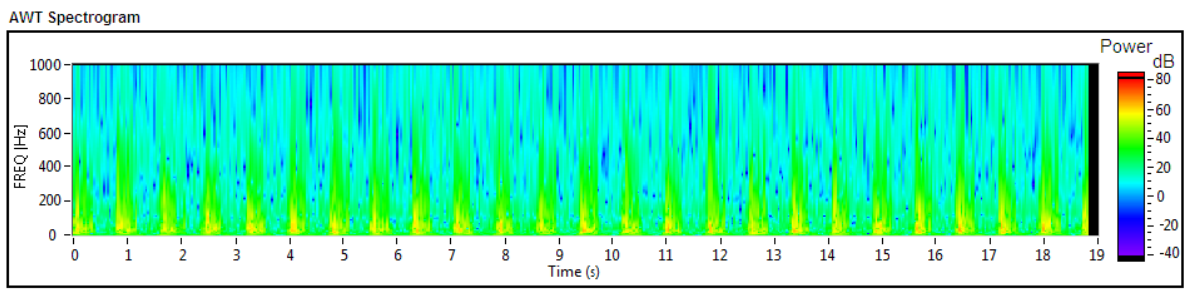

Fig. 3. EMG Wavelet Spectrogram

As for HR, LA and VO2 measures, once the data coming from the test were stored, the data themselves were processed by means of statistical tools. The processing was made in order to evaluate the change of each parameter according to the different positions and intensity levels.

In particular, for the EMG data, for each muscle a 4 (Positions) x 3 (intensity level) x 2 (Groups) ANOVA for repeated measures was applied to verify statistical differences in EMG data expressed as percentages of basal values. After data processing it is useful to analyse correlation between data coming from different devices.

\section{Results}

The data resulting from each test use a lot of storage memory and present a variety of typologies since they are the results of a processing of various signals. As a consequence, computational time to get the information about the quality of a training session is long.

MIF for each single muscle in the three intensity levels in both groups was computed in order to evaluate contraction power variation for each of them, thus underlining fatigue level. In tables 2 and 3 mean values of MIF in the four positions investigated for the different intensities are shown. From EMG's statistical analysis, no difference emerged between groups. 
Table 2. MIF Values for T group at different positions and intensity levels

\begin{tabular}{|c|c|c|c|c|c|c|c|c|c|c|}
\hline & \multicolumn{2}{|l|}{ Muscle } & \multicolumn{2}{|l|}{ VM } & \multicolumn{2}{|c|}{ RF } & \multirow[b]{2}{*}{300} & \multicolumn{3}{|c|}{ BF } \\
\hline & Intensity & 120 & 200 & 300 & 120 & 200 & & 120 & 200 & 300 \\
\hline & $B$ & 53.2 & 46.6 & 46.7 & 77.5 & 52.3 & 49.7 & 70.2 & 51.2 & 45.8 \\
\hline$\stackrel{5}{5}$ & $I$ & 92.3 & 78.6 & 79.1 & 98.5 & 79.7 & 80.1 & 70.3 & 78.0 & 79.3 \\
\hline$\cdot \stackrel{\pi}{\pi}$ & $A$ & 72.3 & 53.4 & 51.6 & 90.2 & 77.8 & 60.2 & 90.6 & 74.3 & 58.1 \\
\hline 2 & $F$ & 80.6 & 61.3 & 59.9 & 78.8 & 60.1 & 61.2 & 92.4 & 70.3 & 71.2 \\
\hline
\end{tabular}

Table 3. MIF Values for UT goup at different positions and intensity levels

\begin{tabular}{|c|c|c|c|c|c|c|c|c|c|c|}
\hline & \multicolumn{2}{|l|}{ Muscle } & \multicolumn{2}{|l|}{ VM } & \multicolumn{2}{|c|}{ RF } & \multirow[b]{2}{*}{300} & \multicolumn{3}{|c|}{ BF } \\
\hline & Intensity & 120 & 200 & 300 & 120 & 200 & & 120 & 200 & 300 \\
\hline & $B$ & 70.5 & 60.2 & 60.1 & 100.1 & 82.2 & 77.6 & 130.1 & 82.0 & 80.0 \\
\hline 5 & $I$ & 82.3 & 73.5 & 72.9 & 99.6 & 81.0 & 80.0 & 82.6 & 78.6 & 70.5 \\
\hline$: \tilde{\Xi}$ & $A$ & 78.6 & 66.4 & 67.0 & 99.8 & 96.5 & 83.4 & 120.8 & 102.3 & 99.0 \\
\hline 2 & $F$ & 60.0 & 47.6 & 51.3 & 71.2 & 48.3 & 54.6 & 98.6 & 120.0 & 140.1 \\
\hline
\end{tabular}

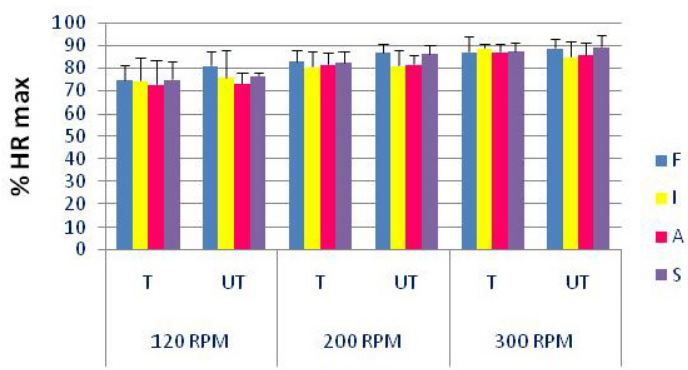

\section{LEVELS}

Fig. 4. Maximum Heart Rate value at different positions and intensity level for $\mathrm{T}$ and UT groups

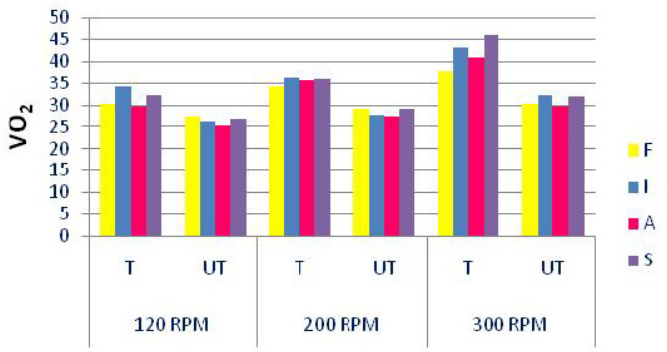

LEVELS

Fig. 5. VO2 mean value at different positions and intensity level for T and UT groups 
About $\mathrm{HR}$ and $\mathrm{VO}_{2}$ we observed a statistically significant difference only during the position $\mathrm{F}$ at the middle intensity among trained and untrained subjects, but the post hoc analysis did not show any significant difference (figure 4 and 5).

We did not find any significant difference for the blood lactate values in both groups.

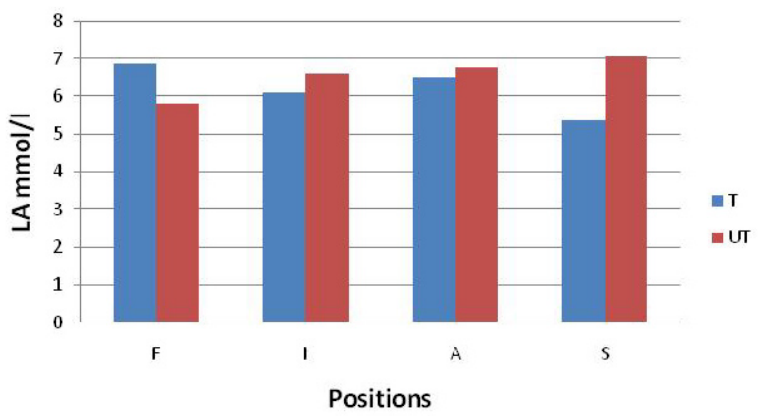

Fig. 6. Mean value of lactate peak at different positions and intensity level for $\mathrm{T}$ and UT groups

\section{Discussion}

On the basis of the results obtained, indications useful for the optimization of the training sessions were found. For instance, we observed an increase of the exercise intensity and muscular activation during the training session in $\mathrm{F}$ position. In UT group there was a trend towards a higher value of MIF, probably due to the lower economy of this intense exercise. However, the lack of statistical significance between T and UT may be attributed to the lower number of subjects.

As above mentioned, the insufficient statistical relevance of the data can also depend on the inefficiency of the whole measuring and processing procedures. These difficulties lengthen measuring and processing times a lot, thus making the monitoring of a high number of subjects difficult.

This problem might be solved by using a structure based on computer grids. In fact, nowadays this technology is very used for similar applications in different research fields 16, 17. (figure 7). Generally speaking, grid computing is the application of several computers to a single problem at the same time. It is usually about scientific or technical problems that require a great number of computer processing cycles or access to large amounts of data.

Ideally, a grid should provide full-scale integration of heterogeneus computing resources of any type: processing units, storage units, communication units, and so on 18.. For this problem a data grid can be used. It has the data storage capacity as its main shared resource. Such a grid can be regarded as a massive data storage system built up from portions of a large number of storage devices. Particularly, a remote user (e.g. a researcher) can manage data coming from different fitness centres and process them by using the grid resources at the same time, which would allow to manage also a much bigger database. Grid could be seen as a way to store data and to 


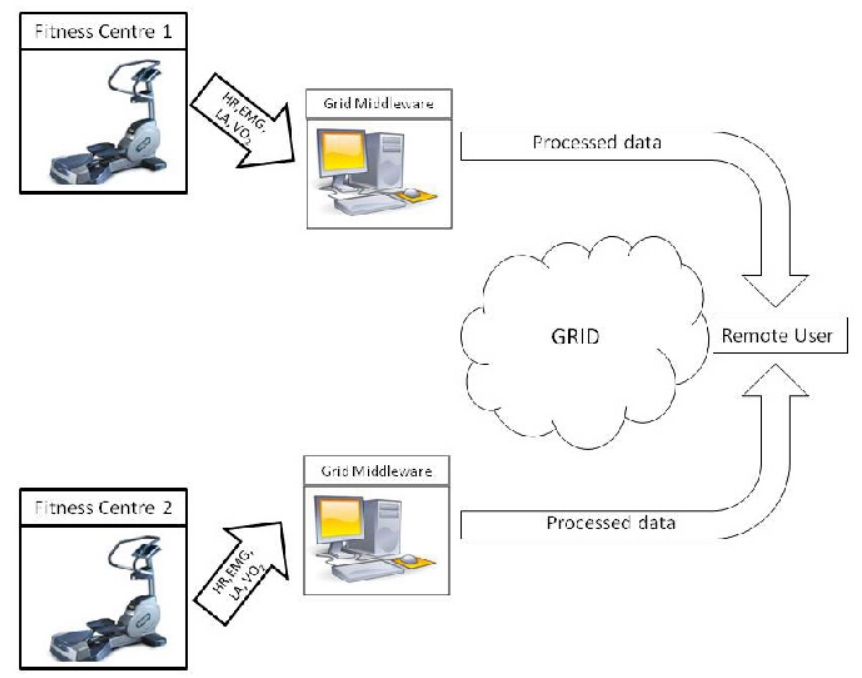

Fig. 7. Scheme of the proposed system on the grid

make them available to multiple users. The computing resources must satisfy basic requirements 18.: They must have enough computing power and data storage capacity to properly run the grid platform; they do not need to be directly connected to each other but they must know some entity that takes it to the grid; they can be indirectly connected through routers, gateways, hubs, switches, bridges, and wireless connections, by which a data packet can be sent from one computing resource to another.

For a data grid, the overall data storage capacity of a grid is the sum of the storage capacity made available for the grid in all its nodes and the performance of a data grid heavily depends on its communication links.

\section{Conclusion}

The paper presented a integrated system to manage and analyze different data in order to evaluate the training in cardiofitness activity. EMG, LA, HR and $\mathrm{VO}_{2}$ were monitored during a training session and stored in a local database. The data were processed and a statistical analysis was performed in order to give usefully information to optimize the training session. Moreover a database containing all the monitored parameters for the different subjects was made. Future work will deal with the application of this system on the grid in order to improve its efficiency, reducing computational times and making the monitoring of a high number of subjects easy.

\section{References}

1. Siscovick, D.S., Weiss, N.S., Fletcher, R.H., Schoenbach, V.J., et al.: Habitual vigorous exercise and primary cardiac arrest: effect of other risk factors on the relationship. J. Chronic. Dis. 37(8), 625-631 (1984) 
2. Rahnama, N., Reilly, T., Lees, A., Graham-Smith, P.: Muscle fatigue induced by exercise simulating the work rate of competitive soccer. J. Sports Sci. 21(11), 933-942 (2003)

3. Enoka, R.M., Stuart, D.G.: Neurobiology of muscle fatigue. J. Appl. Physiol. 72(5), 16311648 (1992)

4. Youdas, J.W., Hollman, J.H., Hitchcock, J.R., Hoyme, G.J., et al.: Comparison of hamstring and quadriceps femoris electromyographic activity between men and women during a single-limb squat on both a stable and labile surface. J. Strength Cond. Res. 21(1), 105-111 (2007)

5. Johnson, J.H., Prins, A.: Prediction of maximal heart rate during a submaximal work test. J. Sports Med. Phys. Fitness 31(1), 44-47 (1991)

6. Kawabata, T., Suzuki, T., Miyagawa, T.: Effect of blood volume on plasma volume shift during exercise. Journal of Thermal Biology 29(7-8), 775-778 (2004)

7. Bandy, W.D., Irion, J.M., Briggler, M.: The effect of static stretch and dynamic range of motion training on the flexibility of the hamstring muscles. J. Orthop. Sports Phys. Ther. 27(4), 295-300 (1998)

8. Hermens, H.J., Freriks, B.: The State of the Art on Sensors and Sensor Placement Procedures for Surface ElectroMyoGraphy: A proposal for sensor placement procedures, Enschede - The Netherlands: Roessingh Research and Development (1997)

9. Kang, J., Chaloupka, E.C., Mastrangelo, M.A., Hoffman, J.R., et al.: Metabolic and perceptual responses during Spinning cycle exercise. Med. Sci. Sports Exerc. 37(5), 853859 (2005)

10. Green, J.M., McLester, J.R., Crews, T.R., Wickwire, P.J., et al.: RPE-lactate dissociation during extended cycling. Eur. J. Appl. Physiol. 94(1-2), 145-150 (2005)

11. Kang, J., Mangine, G.T., Ratamess, N.A., Faigenbaum, A.D., et al.: Influence of intensity fluctuation on exercise metabolism. Eur. J. Appl. Physiol. 100(3), 253-260 (2007)

12. Green, J.M., McLester, J.R., Crews, T.R., Wickwire, P.J., et al.: RPE association with lactate and heart rate during high-intensity interval cycling. Med. Sci. Sports Exerc. 38(1), 167-172 (2006)

13. Dolbow, D.R., Farley, R.S., Kim, J.K., Caputo, J.L.: Oxygen consumption, heart rate, rating of perceived exertion, and systolic blood pressure with water treadmill walking. $\mathrm{J}$. Aging. Phys. Act. 16(1), 14-23 (2008)

14. Kumar, D.K., Pah, N.D., Bradley, A.: Wavelet analysis of surface electromyography to determine muscle fatigue. IEEE Trans. Neural Syst. Rehabil. Eng. 11(4), 400-406 (2003)

15. Ren, X., Yan, Z., Wang, Z., Hu, X.: Noise reduction based on ICA decomposition and wavelet transform for the extraction of motor unit action potentials. J. Neurosci. Methods 158(2), 313-322 (2006)

16. Frizziero, E., Gulmini, M., Lelli, F., Maron, G., et al.: Instrument Element: a new Grid component that enables the control of remote instrumentation. In: Proceedings of the Sixth IEEE International Symposium on Cluster Computing and the Grid Workshops (CCGRIDW 2006). IEEE, Los Alamitos (2006)

17. Lelli, F., Frizziero, E., Gulmini, M., Maron, G., et al.: The many Faces of the Integration of Instruments and the Grid. International Journal of Web and Grid Services 3(3), 239-266 (2007)

18. Costa, S.R.R., Neves, L.G., Ayres, F., Mendonca, C.E., et al.: GridBR: The challenge of grid computing. Grid and Cooperative Computing, pt. 1, 601-607 (2004) 